\title{
AOTrauma European Faculty Seminar 2016
}

Jan Philipp Schüttrumpf

\begin{abstract}
Die AOTrauma Deutschland vergibt jedes Jahr Plätze für das AOTrauma European Faculty Seminar, welches im Rahmen der Davoser AO-Kurse stattfindet. In diesem Seminar wird ausgewählte AO Faculty durch Falldiskussionen weitergebildet. Voraussetzungen für Teilnahme sind die Mitgliedschaft in der AOTrauma, ein erfolgreich absolvierter AOTrauma-Grund- und Fortgeschrittenenkurs sowie die vorausgegangene Aktivität als Faculty bei AO-Veranstaltungen oder ein AO Fellowship. Für die Bewerbung musste ein Patientenfall mit einer Komplikation eingereicht werden. Im Dezember 2016 konnten - ausgewählt nach einer öffentlichen Ausschreibung 3 Ärzte aus Deutschland teilnehmen: Lars Becker (Essen), Jan Philipp Schüttrumpf (Magdeburg) und Panajotis Telisselis (Passau).
\end{abstract}

\section{Erfahrungsbericht von Dr. Jan Philipp Schüttrumpf, Oberarzt der Klinik für Unfallchirurgie des Universitäts- klinikums Magdeburg}

Nach erfolgreicher Bewerbung konnte ich eines der Stipendien der AOTrauma Deutschland ergattern und als Regional Faculty nach Davos, dem Mekka der AO, reisen. Bei herrlichem Sonnenschein und blauem Himmel versammelten wir uns im Kongresszentrum in Davos, wo wir sehr herzlich empfangen wurden. Hier sollten 2 intensive Tage unter der Leitung erfahrener Unfallchirurgen als Faculty folgen (Chairperson: Peter Brink [Niederlande]; Co-Chairpersons: Heiner Winker [Deutschland], Marco Berlusconi [Italien], Jonathan Monk [England]).

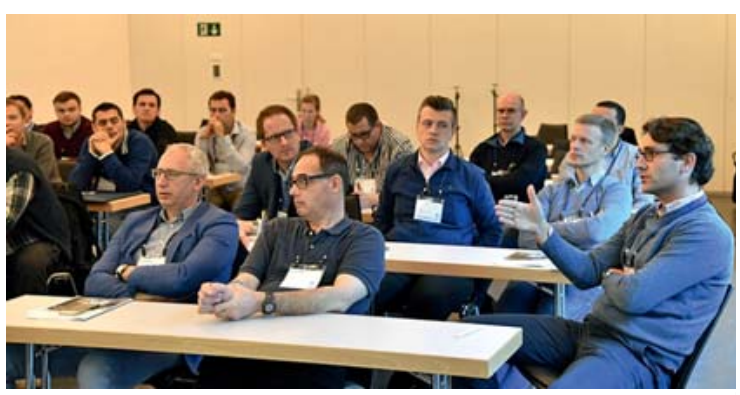

Abb. 1 Diskussion eines Patientenfalls. Bild: AO-Stiftung.
Die 29 Teilnehmer (Regional Faculty) kamen aus ganz Europa. Jeder durfte einen Fall einer gescheiterten oder misslungenen Operation vorstellen unter dem Leitthema „Fracture Complications: When Things Do Not Work out the Way You Thought“. Diese Fälle wurden anschließend in der Gruppe diskutiert. Besonders beeindruckend waren dabei die Vorgehensweisen in den unterschiedlichsten Ländern und die Fehleranalyse sowie die Erarbeitung von Lösungsansätzen in der Gruppe. Immer wieder an die AO-Techniken und deren Vorgehensweisen angelehnt, wurde ehrlich und offen diskutiert. „Aus Fehlern lernen“ - dieses Konzept ist wirklich beeindruckend, und ich habe viele neue Ideen und Techniken für meine alltägliche Praxis als Unfallchirurg mitnehmen dürfen und meinen Horizont erweitern können.

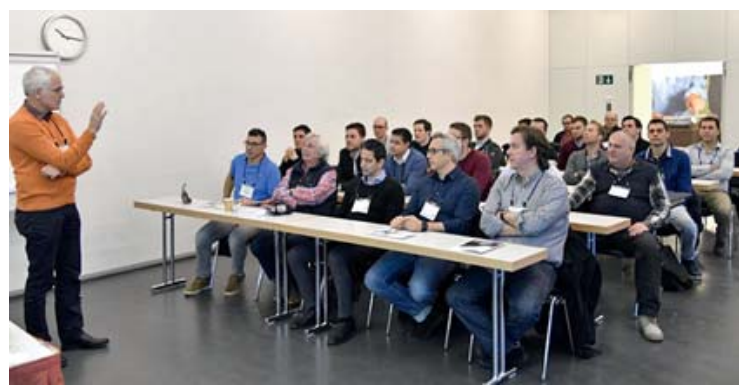

- Abb. 2 Peter Brink als Vortragender. Bild: AO-Stiftung. 


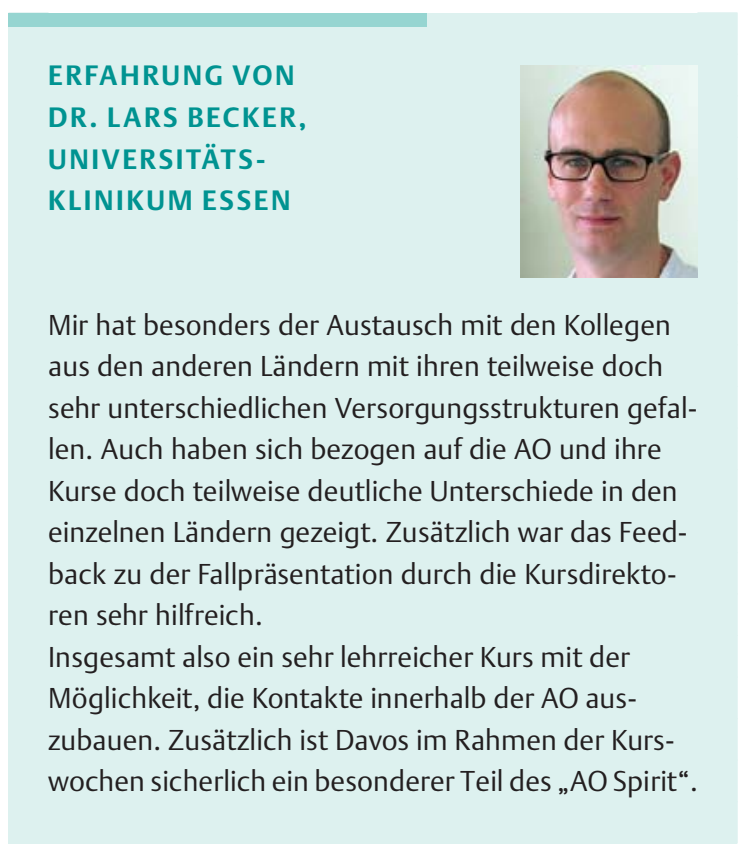

Die 4 besten Fallpräsentationen wurden ausgewählt und mit einer Einladung als Observer Faculty zu einem AO-Basiskurs nach Leeds (England) prämiert. Es freut und ehrt mich wirklich sehr, einer der Glücklichen zu sein und somit den AO-Gedanken weiter leben und auch lehren zu können.

Am Ende des 2. Kurstags stellte sich uns noch das AOTrauma Europe Board vor (Paul Szypryt, Michael Blauth, Steven Velkes und Peter Brink), gab einen Einblick in dessen Aktivitäten und berichtete von laufenden Projekten, aber auch Plänen für die Zukunft.

\section{ERFAHRUNG VON \\ DR. PANAJOTIS TELISSELIS, PASSAU}

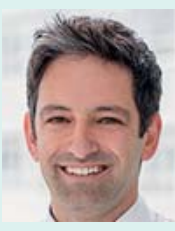

Der Kurs war didaktisch sehr gut aufgebaut und ließ nach der Vorstellung von spannenden „Problemfällen“ aus unserem Alltag genug Raum für teils hitzige Diskussionen. Auf individuelle Beiträge wurde ausreichend eingegangen.

Die flache Hierarchie unter den Teilnehmern ermöglichte ein sehr kollegiales, angenehmes Miteinander. Zahlreiche Teilnehmer aus unterschiedlichsten Ländern trugen zum Facettenreichtum dieser Veranstaltung bei. Man bekam die Möglichkeit, zumindest ein bisschen über den eigenen Tellerrand hinauszusehen.

Es folgte eine Präsentation der neuen E-Learning-Plattform STaRT (surgical training and assessment for residents), einem wirklich beeindruckenden Programm, dass auch bereits eine Auszeichnung erfahren durfte (Winner E-Healtcare Leadership Awards 2014).

Die 2 Tage waren natürlich mehr als nur ein Seminar, denn der Ort Davos mit dem hier vorherrschenden „AO Spirit“ und die neu gewonnenen Kontakte rundeten das Bild ab. Ein gemeinsamer Fondueabend nach dem 1. Seminartag zeigte auf, dass wir als AO-Mitglieder eine gemeinsame Sprache in Europa und auch weltweit sprechen. Hier war auch genügend Zeit für private Gespräche und es wurden neue Freundschaften geschlossen.

\section{AUSSCHREIBUNG VON 2 PLÄTZEN FÜR DAS EUROPEAN FACULTY SEMINAR 2017}

Das AOTrauma Europe Faculty Seminar findet am 2. und 3. Dezember 2017 in Davos statt. Auf dem Faculty Seminar in Davos werden die 4 besten Referenten mit den interesstesten Fällen ausgewählt und zu einem AOTrauma Kurs in das United Kingdom eingeladen.

Die Deutsche AOTrauma wird 2 Plätze für dieses Seminar vergeben, auf die Sie sich ab sofort bewerben können.

Voraussetzungen sind:

- bereits mindestens einmal Referent/Tischinstruktor auf einem AOTrauma Kurs/Seminar,

- Faculty Education Program (FEP) absolviert (von Vorteil, nicht Voraussetzung),

- nicht als Faculty in Davoser AOTrauma Kursen 2017 eingebunden,

- bisher noch nie am AOTrauma Europe Faculty Seminar teilgenommen,

- Mitglied der AOTrauma.

Bewerbungen für einen der beiden Plätze können bis Sonntag, 11. Juni 2017 per E-Mail eingereicht werden an Philip Wilbrandt: p.wilbrandt@aodeutschland.de

Einzusenden sind:

- Kurz-CV mit Nachweis Faculty/Table Instruktor bei AOTrauma-Kursen/-Seminaren

- Fall mit Komplikation (in englischer Sprache) 
Ich möchte mich ganz herzlich bedanken bei Larissa Welti (Project Manager Education and Observations Europe) für die einwandfreie Organisation, bei der Faculty des European Faculty Seminars unter der Leitung von Peter Brink für die lehrreichen Tage, bei den Kollegen aus ganz Europa für die inspirativen Fallvorstellungen und bei der AOTrauma Deutschland für die Möglichkeit, an diesem exzellenten Seminar teilnehmen zu können.

Über die Autoren

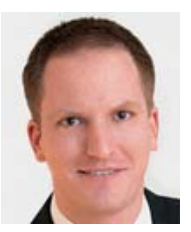

Korrespondenzadresse

Dr. med. Jan Philipp Schüttrumpf

Oberarzt der unfallchirurgischen Klinik

Universitätsklinikum Magdeburg A. ö. R.

Leipziger Str. 44

39120 Magdeburg

jan.schuettrumpf@med.ovgu.de

Bibliografie

DOI https://doi.org/10.1055/s-0043-109132

OP-JOURNAL 2017; 33: 87-89 @ Georg Thieme Verlag KG Stuttgart · New York ISSN 0178-1715 